\title{
De l'étatique au local : le New Labour, l'individu et les valeurs de la communauté
}

From the State to the Local Level: New Labour, the Individual and Community Values

Jérôme Tournadre-Plancq

\section{OpenEdition} Journals

Édition électronique

URL : http://journals.openedition.org/rfcb/3404

DOI : $10.4000 /$ rfcb.3404

ISSN : 2429-4373

Éditeur

CRECIB - Centre de recherche et d'études en civilisation britannique

Édition imprimée

Date de publication : 1 avril 2005

ISBN : 2-911580-20-6

ISSN : 0248-9015

Référence électronique

Jérôme Tournadre-Plancq, « De l'étatique au local : le New Labour, l'individu et les valeurs de la communauté », Revue Française de Civilisation Britannique [En ligne], XIII-2 | 2005, mis en ligne le 01 avril 2005, consulté le 10 avril 2020. URL : http://journals.openedition.org/rfcb/3404 ; DOI : https:// doi.org/10.4000/rfcb.3404

Ce document a été généré automatiquement le 10 avril 2020.

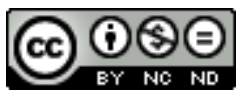

Revue française de civilisation britannique est mis à disposition selon les termes de la licence Creative Commons Attribution - Pas d'Utilisation Commerciale - Pas de Modification 4.0 International. 


\section{De l'étatique au local : le New Labour, l'individu et les valeurs de la communauté}

From the State to the Local Level: New Labour, the Individual and Community Values

Jérôme Tournadre-Plancq

1 Des divers procès faits au néo-travaillisme depuis le milieu des années 1990, le plus régulièrement instruit demeure celui consistant à dépeindre son projet politique, la "Troisième voie ", comme un "simple vol des habits de la droite» destiné, selon le néoconservateur états-unien Francis Fukuyama, à "défendre le noyau de la révolution thatchérienne $»^{1}$. Le discours de la réforme inscrit au cœur de ce "néo-progressisme $»^{2}$ alimente ainsi, en s'éloignant des positions traditionnelles - ou perçues comme telles du travaillisme classique, une critique prompte à le présenter comme un simple " thatchérisme à visage humain $\|^{3}$. Ce discours légitimerait, notamment, le retrait de l'État de ses différentes sphères d'influence et de compétence, tant politiques, qu'économiques ou sociales. Au-delà d'évolutions institutionnelles notables (mise en place d'une assemblée élue au Pays de Galles et d'un parlement en Écosse), la victoire des travaillistes en mai 1997 a en effet amorcé un mouvement plus général et diffus de valorisation du local au détriment de l'étatique. Comme le souligne Gordon Brown, la décentralisation doit ainsi dépasser la «dévolution régionale et locale du gouvernement [et] implique de déléguer le pouvoir du gouvernement...vers les communautés et voisinages locaux" ${ }^{4}$. Le discours de légitimation de ce mouvement avance par conséquent que l'État ne doit plus être exclusivement appréhendé comme un " propriétaire ou un employeur » mais comme un "partenaire, un habilitateur, un catalyseur, un coordinateur ${ }^{5}$. Pensée en rupture avec un travaillisme tenu à l'écart de l'exercice du pouvoir central entre 1979 et $1997^{6}$, la Troisième voie se donne dès lors à voir comme un remède à la propension centralisatrice $d u$ thatchérisme et, surtout, d'un "mid-century fabianism » ${ }^{7}$ que résumerait le fameux mot de Douglas Jay : «[t]he men of Whitehall know best ». 
Ce repositionnement résulte également du rapport de forces interne caractérisant le Parti travailliste "pré-Troisième voie $\|^{8}$. Face à une aile gauche puissante qu'ils accusent d'être à l'origine de la perte de crédit électoral du parti, des membres du centre et de la droite travaillistes entreprennent ainsi de façonner un discours susceptible de se poser en alternative dans l'espace politique britannique des années 1980. Des universitaires et certains parlementaires s'engagent dans une redécouverte des racines idéalistes, chrétiennes et libérales du progressisme britannique ${ }^{9}$. Cette entreprise conduit à la production d'un discours essentiellement structuré autour des notions de "communauté », de "responsabilisation », de "citoyenneté », et intéressé par les rapports existant entre le socialisme et l'exercice de la participation individuelle à la vie de la société; un discours qui nourrit celui de Neil Kinnock, leader de 1983 à 1992, et de ceux rangés sous la bannière de la «modernisation ». Un document rédigé par l'universitaire Bernard Crick et le parlementaire David Blunkett ${ }^{10}$, futur ministre des gouvernements Blair, et relatif aux «buts et valeurs » du travaillisme est significatif de cette nouvelle philosophie. Il expose en effet la nécessité de lier la pleine réalisation d'une société égalitaire et solidaire à celle d'une société démocratique dans laquelle, le gouvernement central ayant fixé des lignes de conduite et des normes nationales, il revient aux régions, aux localités et aux communautés de participer et d'exercer par là même leur liberté.

3 Si la Troisième voie se voit régulièrement reprocher par ses détracteurs et contradicteurs de n'être qu'un simple opportunisme électoral chevillé à un solide pragmatisme $^{11}$, il n'en demeure pas moins que celle-ci a, au fil de sa phase d'énonciation et de conceptualisation, donné lieu à la constitution d'un espace de théorisation quasi-autonome vers lequel convergent divers entrepreneurs intellectuels, de l'universitaire reconnu au think tank "passeur " d'idées ${ }^{12}$. C'est dès lors la Troisième voie en tant que produit de cet espace de théorisation - et non en tant qu'ensemble de politiques publiques - qui constituera notre objet d'étude tout au long de cet article. Notre ambition consistera en effet à souligner que, quelle que soit l'interprétation que l'on puisse lui donner, le mouvement de décentralisation néo-travailliste s'appuie sur des principes théoriques clairement énoncés dans le corps de doctrine que constitue, à notre sens, la Troisième voie. En entendant fonder une "nouvelle relation entre l'individu, les communautés et le gouvernement ${ }^{13}$, il se veut une réponse aux demandes liées à l'individualisation croissante de la société et le support d'une nouvelle conception de l'égalité. Ce mouvement se donne en outre à voir comme le plus sûr chemin vers l'émergence d'une "société civique forte »"

\section{S'adapter au « nouvel individualisme »}

4 Située aux confluents des discours respectifs tenus par les néo-travaillistes sur la question sociale, l'émancipation politique et la réforme des institutions, l'idée même de décentralisation s'inscrit, si l'on en croit Tony Blair, dans l'ordre des choses. "[Les] normes nationales [étant] nécessaires, mais pas suffisantes"”, il s'avère en effet indispensable de "franchir une nouvelle étape de décentralisation » en répondant au mieux " aux besoins locaux et individuels " $^{15}$. Cette mise en avant de l'individu et de ses besoins et revendications rappelle combien la Troisième voie est avant tout, et peut-être surtout, un projet politique post-fordiste. Ce "néo-progressisme» entend en effet rompre avec la lecture de la société ayant prévalu en Occident au lendemain du second conflit 
mondial ; une lecture où s'entrecroisent production de masse, homogénéité du salariat et division sexuée et classiste de l'ordre social. Les structures nées de cette approche de la société et imposant « un schéma unique pour tous ${ }^{16}$ sont en effet contestées dans leur légitimité et leur efficacité par l'émergence de ce que les sociologues Anthony Giddens et Ulrich Beck, acteurs essentiels de l'espace de théorisation de la Troisième voie, qualifient de «nouvel individualisme ».

5 Cet individualisme, caractéristique de la période de «modernité avancée » et globalisée que traversent les sociétés contemporaines, ne se réduit pas, contrairement à son pendant néo-libéral, au consumérisme et à une maximisation des seuls intérêts individuels. Il ne renvoie pas, non plus, à l'atomisation et à l'isolement ${ }^{17}$. Il s'inscrit dans la logique d'une ère " post-traditionnelle $»^{18}$, conduisant à la remise en cause de tout ce que l'homme tenait pour des vérités incontestées et qui, jusqu'alors, conditionnait son existence. Désormais débarrassés de ces carcans uniformisants et, par conséquent, libres d'adopter les styles de vie et les conduites individuelles qu'ils désirent, les "gens sont invités à se constituer en tant qu'individus $»^{19}$, à opérer des choix, tout en produisant ensemble leur propre "biographie $»^{20}$. L'autonomisation des individus n'est en effet rendue possible que par la forte interdépendance sociale liant la société ou, plus exactement, la communauté. Cette dernière renvoie à ces espaces - la Nation, le voisinage, la famille, etc... - au sein desquels chacun est amené à se construire. Comme le souligne Gordon Brown, «les pouvoirs de la communauté [doivent ainsi] permettre aux gens de prendre le contrôle de leur propre vie $»^{21}$ et faire de cet individualisme un « individualisme collectif $»^{22}$.

6 Ce phénomène "d'invidualisation ${ }^{23}$ est source de décalages entre les politiques définies par l'État central et les préoccupations des citoyens au niveau local. Elle implique donc, selon les partisans de la Troisième voie, un transfert du pouvoir au plus près de ces citoyens. Une telle configuration peut, entre autres, conduire à adopter ce que le socialiste belge Franck Vandenbroucke décrit, dans une revue proche du pouvoir néotravailliste, comme étant une "approche sur mesure " ('tailor-made approach') ${ }^{24}$ : une approche ne se pensant plus en termes de groupes sociaux mais se définissant, notamment dans la fourniture de prestations tant sociales que politiques, par une adaptation à l'hétérogénéité des situations individuelles. Comme le souligne - dans un autre contexte - Pierre Rosanvallon, ce ne sont plus désormais «les identités collectives qu'il faut décrire, mais des parcours individuels ${ }^{25}$. Cette approche peut caractériser un État réformé, traitant de manière plus déconcentrée et sensible des questions relevant de ses domaines d'intervention. L'une des illustrations les plus significatives de cette démarche réside ainsi dans la tâche dévolue aux Job Centers, agences locales censées fournir aux individus à la recherche d'un emploi les moyens personnalisés, notamment en termes de formation, de trouver leur place sur le marché de l'emploi. Cette approche doit également susciter la constitution de partenariats divers et décentralisés dont la caractéristique résiderait, selon les néo-travaillistes, dans leur flexibilité et leur capacité d'adaptation aux individualités; on pense, par exemple, aux partenariats appelés de ses vœux par Tony Blair entre les collectivités locales, les écoles et les parents afin de mettre en place des programmes ciblés de lutte contre l'analphabétisme ${ }^{26}$. 


\section{La décentralisation et l'égalité}

7 L'approche décrite précédemment n'est pas sans liens avec le travail de réflexion sur l'égalité fourni par les divers contributeurs au débat sur la Troisième voie. L'égalité et la place qu'elle occupe dans le corpus théorique de ce projet politique constituent en outre le point de ralliement de divers courants critiques producteurs d'une lecture disqualifiante du néo-travaillisme en tant que projet politique de centre gauche. Anthony Giddens, sociologue régulièrement présenté comme « l'intellectuel préféré de Tony Blair » et le principal théoricien de la Troisième voie, considère pourtant qu'un discours de gauche exprimant un désintérêt, même relatif, pour l'égalité s'avèrerait à terme intenable ${ }^{27}$. Cette valeur doit, de ce fait, structurer une part importante de la réflexion sur le "renouveau de la social-démocratie ». Les thirdwayers entendent avant tout rompre avec une égalité de résultats qu'ils associent au "vieux travaillisme " ('old Labour'),

[Non] parce que [cette conception de l'égalité] est trop radicale, mais parce que ce n'est ni désirable ni faisable [...]. Les résultats prédéterminés et imposés [...] par une autorité centrale et décidés sans prise en compte du travail, des efforts ou de la contribution à la communauté ne sont pas un rêve socialiste mais le cauchemar que certains font du socialisme [...]. Cela nie toute humanitée ${ }^{28}$.

Est par conséquent préférée à cette égalité de résultats une égalité réelle des chances (' Real Equality of Opportunity'), distincte, selon ses promoteurs, de la formelle égalité des chances libérale. Associée au respect de la pluralité des choix et besoins individuels, l'égalité réelle des chances aurait pour vertu d'attaquer les inégalités à leur racine, non par l'intermédiaire d'une "uniforme» et "impersonnelle " redistribution matérielle accusée de reproduire un schéma où le sommet décide pour la base de la société ('topdown'), mais en assurant l'accès de tous à ces biens préventifs et stratégiques - les ' opportunity goods' - que sont la formation, l'éducation, l'acquisition de compétences et qualifications, la culture, le travail ou le pouvoir politique. La puissance publique a en effet pour tâche d'habiliter les individus, de les rendre capables (to enable) de saisir les opportunités de trouver pleinement leur place dans la société. Gordon Brown affirme ainsi explicitement que l'enjeu est désormais, au travers de la défense de cette conception de l'égalité, de permettre aux individus de se réaliser pleinement, de " combler le fossé entre ce qu'ils sont et ce qu'ils peuvent potentiellement devenir ${ }^{29}$. C'est en cela que la décentralisation, et la 'tailor-made approach' qu'elle induit, peuvent servir l'égalité. Elle doit permettre aux opportunities de se propager de manière parfaite et adéquate dans la société et, au risque de se détacher d'un universalisme socialdémocrate, prendre en compte les disparités et réalités locales afin de réaliser ce qu'Alan Milburn, architecte du prochain manifeste électoral néo-travailliste, conçoit comme la "cause progressiste moderne $»^{30}$.

9 La situation découlant de l'égal accès de tous aux opportunity goods ne constitue pas une fin en soi pour les partisans et théoriciens de la Troisième voie. Elle doit en effet permettre d'atteindre ce qui, aux yeux de Tony Blair, constitue «l'essence de la justice sociale $~^{31}$ : La naissance en chacun d'un sentiment d'appartenance au tout social résidant dans l'extension à tous d'un intérêt (stake) dans l'activité sociale. C'est en grande partie sur ce fondement qu'est redéfinie la notion de citoyenneté. 


\section{Une citoyenneté active et responsable}

10 Comme le rappelle l'universitaire Geoff Andrews, "la citoyenneté a émergé comme une échappatoire idéologique pour la droite et la gauche [de la fin des années 1980] „"2. La première y vit le moyen, au travers du concept d'active citizen développé par Douglas Hurd, de signifier la fin d'un discours thatchérien où seul le marché importait. La seconde put, de son côté, trouver dans cette notion matière à dénoncer les attaques de l'État centraliste conservateur contre les droits civiques. L'énonciation de la Troisième voie, dès la première moitié des années 1990, s'inscrit également dans cette réflexion, la croisant, notamment, avec celle relative à la communauté, espace de décentralisation par excellence. Centrale dans le discours néo-travailliste, la notion de communauté ne traduit pas, comme nous l'avons précédemment exposé, un repli communautaire.

11 Sa présence dans le discours néo-travailliste revêt, comme l'a souligné Laurent Bouvet, l'intérêt stratégique de "toucher une partie de l'électorat conservateur, soucieux avant tout de l'unité sociale du pays [...] tout en répondant à l'une des attentes traditionnelles de l'électorat travailliste, soucieux de solidarité communautaire $»^{33}$. Cet intérêt pour la communauté est souvent interprété comme l'assimilation par les néo-travaillistes de la philosophie communautarienne états-unienne. Il est vrai que le courant «modernisateur » du Labour entretient des relations privilégiées avec son pendant démocrate américain, ce dernier ayant fait sien un discours communautarien mêlant rappels de "devoirs" envers la communauté et éloges du contrôle social. Amitaï Etzioni, sociologue et passeur de la pensée communautarienne du champ académique au champ politique états-unien, a vu ses essais «importés » dans le débat public britannique par le think tank Demos, notoirement proche du New Labour, et est de ce fait perçu comme une influence majeure de la Troisième voie ${ }^{34}$.

12 Toutefois, si, comme dans la pensée communautarienne, l'évocation des responsabilités et devoirs envers la communauté est omniprésente dans le discours néo-travailliste, la différence fondamentale tient, à notre sens, au fait que ce discours est, bien plus explicitement que celui des communautariens états-uniens, intimement lié à la refondation de la citoyenneté. Il entend en effet promouvoir ce que Gordon Brown décrit comme étant une "citoyenneté responsable ». Celle-ci repose sur une idée, déjà présente dans l'œuvre du philosophe Thomas Hill Green, selon laquelle tous les citoyens doivent participer de façon pleine et entière à la vie sociale ; une idée que l'on retrouve dans les écrits de l'un des élèves de Green, Leonard Hobhouse, principal concepteur, au début $\mathrm{du} \mathrm{XX}^{\mathrm{e}}$ siècle, d'un libéralisme social dont les thirdwayers se présentent souvent en héritiers ${ }^{35}$.

13 La figure de l'entrepreneur social, omniprésente dans le premier discours que Tony Blair prononce en 1997 en tant que Premier ministre ${ }^{36}$, épouse au plus près cette définition de la citoyenneté dans le domaine du Welfare et de la décentralisation de ce dernier. Incarnation du leader local idéal dans le discours de la Troisième voie et représentation ultime du citoyen qu'il entend promouvoir, l'entrepreneur social se situe aux frontières communes des secteurs public, privé et tiers et en synthétise les valeurs respectives. Sens de l'intérêt général, opportunisme éclairé et esprit d'innovation, entre autres, alimentent ainsi cet "individualisme créatif ${ }^{37}$ que décrit notamment, dans un document du think tank Demos, Charles Leadbeater ${ }^{38}$, conseiller de Tony Blair. Individualiste tout en se battant pour les intérêts de la communauté, charismatique, doté d'une expertise locale, briseur de règles et de conventions 
contraignant l'action, l'entrepreneur social se donne à voir comme l'anti-thèse du civil servant et permet par là même aux penseurs de la Troisième voie de stigmatiser l'inertie bureaucratique d'un État social présenté comme inadapté aux maux de la société contemporaine. Parce qu'il s'inscrit dans le mouvement amorcé par le "nouvel individualisme ", l'entrepreneur social doit devenir l'agent d'un "type moderne de welfare $d u X X I^{e}$ siècle ${ }^{39}$ en répondant à des besoins locaux jusqu'alors non satisfaits. L'entrepreneur social se caractérise par sa capacité à mobiliser au niveau local des ressources jusqu'alors non exploitées ou sous-exploitées par l'État ou toute autre autorité. Charles Leadbeater illustre ainsi son propos au travers, notamment, de l'exemple d'Andrew Mawson qui, au début des années 1980, fédéra les habitants d'une zone défavorisée de l'Est londonien autour de projets de transformation en nursery, en crèche, en espaces d'activités artistiques et en centre de santé de bâtiments appartenant à l'Église réformée. D'une manière plus générale, la mobilisation de ces ressources - hommes et biens immobiliers - lui permet de créer du capital social au sens où l'entend le sociologue américain Robert Putnam : l'ensemble des relations entre individus et les réseaux sociaux et normes de réciprocité et de confiance qui en émanent ${ }^{40}$. Cette production du capital social permet par conséquent de consolider les communautés concernées ('community-building'), de rassembler les citoyens autour de «valeurs partagées » et, par voie de conséquence, de lutter contre l'exclusion sociale.

\section{L'émergence d'un « Nouveau mutualisme »}

14 Si les partisans de la Troisième voie appréhendent l'entrepreneur social comme un héraut de la modernité, la mise en avant de la citoyenneté et de la "société civique» dans leurs réflexion et rhétorique les conduit également à revendiquer la filiation entre leur projet politique et l'un des piliers du patrimoine socialiste et ouvrier britannique : le mutualisme. Celui-ci fait en effet son retour, porté par certains individus proches d'une tradition chrétienne du socialisme, à la fin des années 1980, avant d'être repris par les principales figures du néo-travaillisme. Frank Field ${ }^{41}$, ministre de la Réforme du Welfare entre mai 1997 et juillet 1998, David Green, ancien labour councillor occupant désormais des responsabilités au sein du think tank très libéral, l'Institute of Economic Affairs, ou Stephen Pollard, ancien conseiller de Tony Blair et membre de la Social Market Foundation, autre think tank, en appellent ainsi au retour d'un pan entier de l'histoire de leur mouvement :

Bien avant le temps de la planification étatique d'après-guerre, et même de la clause IV, les socialistes britanniques étaient reconnus pour leur identification aux principes de fraternité communautaire, de coopération mutualiste, de responsabilité individuelle et d'entraide. Les coopératives, les friendly societies et les syndicats formaient ensemble un réseau de welfare dédié à la fourniture [...] d'efficaces [...] services de santé et de protection sociale à leurs membres ${ }^{42}$.

Comme le rappelle l'historien Edward Thompson, en 1963, dans son ouvrage désormais classique La Formation de la classe ouvrière anglaise ${ }^{43}$, la fin du XVIII siècle fut marquée par la constitution, au sein du monde ouvrier, d'associations corporatistes - les friendly societies et les self-help groups - où se mêlaient le politique et le social: religion, méthodisme, chartisme et owenisme. Fortement marquées par l'autodiscipline et le contrôle social, elles fournirent à la classe ouvrière naissante les moyens d'une " indépendance collective» en mutualisant les risques de maladie, de chômage et en fournissant divers services à leurs membres ${ }^{44}$. 
16 $\mathrm{XX}^{\mathrm{e}}$ siècle, furent marginalisées par l'offre sociale étatique née du vote du National Insurance Act en 1911 puis de l'édification du Welfare State au cours des années 1940. La recherche de l'autonomie individuelle, de la liberté de choix et «l'altruisme naturel $»^{45}$ de chacun, d'une part - la nécessité de trouver des cadres d'exercice de la citoyenneté active, d'autre part - légitiment de renouer avec les fondamentaux de ce mouvement. Si cette réflexion s'appuie sur un appel pour le moins convenu au tiers secteur et au voluntary sector, jugés plus enclins que l'État à l'attention individuelle, elle conduit également les néo-travaillistes à tenir un discours pour le moins inédit en direction des syndicats. Ces derniers, il est vrai, héritiers directs des friendly societies, doivent figurer parmi les premiers récepteurs de la décentralisation en s'érigeant en fers de lance du " nouveau mutualisme $»^{46}$. conservateurs des années $1980^{47}$, passe, selon les partisans de la Troisième voie, par la constitution d'un maillage de structures locales et mutualistes offrant à leurs adhérents des services diversifiés et personnalisés. Ces derniers, relevant jusqu'alors pour certains de la puissance publique, seraient principalement liés au conseil, à l'assurance, à la mise en place de fonds de retraite et de santé, à la direction d'hôpitaux, etc. Cette gestion par les adhérents et pour les adhérents met dès lors en adéquation "décentralisation", «démocratisation» et «transparence». Toutefois, l'avenir syndical esquissé par des néo-travaillistes persuadés, à l'instar de Peter Mandelson et Roger Liddle, que les syndicats n'ont "d'autre alternative que de changer ${ }^{48}$, n'est pas vierge de considérations stratégiques. Il traduit le repositionnement des travaillistes vis-à-vis des organisations syndicales. En restreignant l'avenir du syndicalisme à la sphère sociale, la Troisième voie le détourne fort logiquement d'une activité politique où il a pu, par le passé, gêner les ambitions électorales des travaillistes ${ }^{49}$. En outre, comme le souligne l'universitaire «libertarien » Nigel Ashford ${ }^{50}$, de telles évolutions offrent aux néo-travaillistes le moyen de battre en brèche l'opposition des organisations syndicales aux privatisations en faisant des premières les principales bénéficiaires des secondes.

Le mouvement de décentralisation que porte la Troisième voie se fonde donc sur une certaine lecture de la société ; une société où prédominent la diversité et le risque de fragmentation. Ce transfert de pouvoir semble dès lors satisfaire une double logique. Il permet, d'une part, de répondre à de nouvelles attentes en termes de démocratie et augure par là même, pour reprendre les termes d'Anthony Giddens, d'une « démocratisation de la démocratie " $^{51}$. Il conduit, d'autre part, à la responsabilisation d'un niveau local doté de nouvelles missions et compétences. Ce faisant, la décentralisation néo-travailliste, accusée de ne masquer qu'un simple et opportuniste désengagement de l'État, se traduit essentiellement par l'émergence d'un système de gouvernance tirant sa légitimité de la mise en avant de l'engagement civique de ses acteurs. 


\section{BIBLIOGRAPHIE}

BENTLEY, Tom \& WILSDON, James. The Adaptative State : Strategies for Personalising the Public Realm. London: Demos, 2003.

BLAIR, Tony. Leading the Way : A New Vision for Local Government. London: Institute for Public Policy Research, 1998.

BLAIR, Tony. « Ma vision pour la Grande-Bretagne ». Le Débat, n 124, mars-avril 2003, pp. 53-57.

BLAIR, Tony. « Comment réformer les services publics ? ». En Temps réel, Cahier 9, juin 2003.

BLEARS, Hazel. Communities in Control : Public Services and Local Socialism. London: Fabian Society, 2003.

BRICKELL, Paul. People before Structures : Engaging Communities effectively in Regeneration. London: Demos, 2000.

BROWN, Gordon. 'The Politics of Potential : A New Agenda for Labour' in David Miliband (ed.), Reinventing the Left. Cambridge: Polity Press, 1994.

BROWN, Gordon. 'Civic Society in Modern Britain', 17 th Arnold Goodman Charity Lecture, 20 July 2000.

CHRISTIE, Ian \& LEADBEATER, Charles. To our Mutual Advantage. London: Demos, 1999.

GIDDENS, Anthony. The Third Way: The Renewal of Social Democracy. Cambridge: Polity Press, 1998.

HARGREAVES, Ian. New Mutualism : In from the Cold. The Co-operative Revival and Social Exclusion, London: The Co-operative Party, March 1999.

LEADBEATER, Charles. The Rise of the Social Entrepreneur. London: Demos, 1997.

LEADBEATER, Charles. Personalisation through Participation. London: Demos, 2004.

MILBURN, Alan. 'Localism : The Need for a New Settlement'. Speech at Demos Seminar, 21 January 2004.

\section{NOTES}

1. Francis FUKUYAMA, 'Is there a Worldwide Conservative Crack-Up?', The Weekly Standard, 25 August-1 September 1997, pp. 18-19.

2. Anthony GIDDENS, 'Neoprogressivism: A New Agenda for Social Democracy', in Anthony GIDDENS (ed.), The Progressive Manifesto, Cambridge: Polity Press/Policy Network, 2003, p. 1.

3. La paternité de cette expression, rapportée par le journaliste Peter Hetherington (Peter HETHERINGTON, 'Strong Blair escapes voters', The Guardian, 26 September 1998, p. 9), reviendrait paradoxalement à un électeur conservateur. Elle a depuis fait les beaux jours des observateurs critiques du «blairisme ». (Voir notamment, pour la part française de ces critiques, l'article de Philippe MARLIÈRE, «Un thatchérisme à visage humain », Les Temps modernes, n 601, 1999, pp. 133-151.)

4. Gordon BROWN, 'Civic Society in Modern Britain', 17 th Arnold Goodman Charity Lecture, 20 July 2000.

5. Gordon BROWN, 'Foreword', in Colin CROUCH \& David MARQUAND, Reinventing Collective Action, Oxford: Blackwell/The Political Quarterly, 1995, p. 4. 
6. Les travaillistes se sont donc, par la force des choses, cantonnés à l'exercice du pouvoir local, ce qui tend notamment à expliquer les fortes influence et présence d'élus locaux au sein du groupe parlementaire et du gouvernement Blair.

7. Concernant les attaques et critiques à l'encontre de ce courant majeur du progressisme, voir le document de David PIACHAUD, What's wrong with Fabianism?, Fabian Pamphlet 558, London: Fabian Society, 1993, 19 p.

8. Voir à ce sujet l'ouvrage de Geoffrey FOOTE, The Labour Party's Political Thought: A History, New York: St Martin's Press, 3e édition, 1997 (1985), et plus particulièrement les pages 324 à 348, consacrées à l'émergence du New Labour.

9. Nous évoquerons ainsi, à titre d'exemples significatifs, les ouvrages de : Andrew VINCENT \& Raymond PLANT, Philosophy, Politics and Citizenship, Cambridge: Blackwell, 1984 ; Bryan GOULD, Socialism and Freedom, London: Jonathan Cape, 1989; Raymond PLANT, Citizenship, Rights and Socialism, London: Fabian Society, Fabian Tract 531, 1988 ; Roy HATTERSLEY, Choose Freedom: The Future for Democratic Socialism, London: Michael Joseph, 1987.

10. David BLUNKETT \& Bernard CRICK, The Labour Party's Aims and Values: An Unofficial Statement, London: Spokesman Pamphlet 87, 1988.

11. Voir à ce sujet l'article très critique de l'universitaire David Marquand pourtant intéressé, durant un temps, par l'entreprise de refondation de la social-démocratie que proposait le New Labour: David MARQUAND, "Le Nouveau Travaillisme ou les ambiguïtés de la modernité », Pouvoirs, $n^{\circ}$ 93, 2000, pp. 7-20.

12. La constitution de cet espace de théorisation s'appuie essentiellement sur le fait que Tony Blair a explicitement, dès son arrivée à la tête du Labour en 1994, demandé au «monde des idées » de penser la Troisième voie. Il a, par là même, externalisé la refondation programmatique de l'organisation partisane.

13. Gordon BROWN, 'Civic Society in Modern Britain', op. cit.

14. Tony BLAIR, 'The Strong Society: Rights, Responsibilities and Reform', Speech at Newport, 30 May 2001.

15. Tony BLAIR, « Comment réformer les services publics », En temps réel, Cahier 9, juin 2003, p. 7.

16. Ibid., p. 10.

17. Comme l'avance Anthony Giddens, '[...] the new individualism [...] does not signal a process of moral decay. Rather to the contrary, surveys show that younger generations today are sensitized to a greater range of moral concerns than previous generations were. They do not, however, relate these values to tradition, or accept traditional forms of authority as legislating on questions of lifestyle. Some such moral values are clearly post-materialist in [Ronald] Ingelhart's sense, concerning for example ecological values, human rights or sexual freedom' (Anthony GIDDENS, The Third Way: The Renewal of Social Democracy, Cambridge: Polity Press, 1998, p. 36).

18. Anthony GIDDENS, Beyond Left and Right : The Future of Radical Politics, Cambridge: Polity Press, 1994, p. 5.

19. Ulrich BECK, 'The Cosmopolitan Manifesto', The New Statesman, 20 March 1998, p. 28.

20. Ulrich BECK, 'The Reinvention of Politics : Towards a Theory of Reflexive Modernisation', in Ulrich BECK, Anthony GIDDENS \& Scott LASH, Reflexive Modernization : Politics, Traditions and Aesthetics in the Modern Social Order, Cambridge: Polity Press, 1994, p. 13.

21. Gordon BROWN, Fair is Efficient : A Socialist Agenda for Fairness, London: Fabian Pamphlet 563, 1994, p. 25.

22. Tony WRIGHT, Who wins dares: New Labour - New Politics, London: Fabian Society/The New Statesman, 1997, p. 6.

23. Ulrich BECK, «Le conflit des deux modernités et la question de la disparition des solidarités », Lien social et Politiques, $\mathrm{n}^{\circ}$ 39, printemps 1998, p. 21.

24. Franck VANDENBROUCKE, ‘Active Welfare’, Policy Network, 1, Spring 2001, p. 139. 
25. Pierre ROSANVALLON, La nouvelle question sociale, coll. « La couleur des idées », Paris : Le Seuil, 1995, p. 209.

26. Tony BLAIR, Leading the Way: A New Vision for Local Government, London: Institute for Public Policy Research, 1998, p. 7.

27. Voir notamment le désormais classique essai d'Anthony GIDDENS, The Third Way: The Renewal of Social Democracy, op. cit., 166 p.

28. '[...] We reject [this conception of equality] not because it is too radical but because it is neither desirable nor feasible [...]. Pre-determined results imposed [...] by a central authority and decided irrespective of work, effort or contribution to the community, it is not a socialist dream but other people's nightmare of socialism [...] It denies humanity' (Gordon BROWN, 'Equality - Then and now', in Dick LEONARD (ed.), Crosland and New Labour, Basingtoke: Macmillan/Fabian Society, 1999, p. 42).

29. Gordon BROWN, 'The Politics of Potential : a New Agenda for Labour', in David MILIBAND (ed.), Reinventing the Left, Cambridge: Polity Press, 1994, p. 113.

30. Alan MILBURN, 'Localism : The Need for a New Settlement', Speech at Demos Seminar, 21 January 2004.

31. Tony BLAIR, New Britain : My Vision of a Young Country, London: Fourth Estate, 1996, p. xi.

32. Geoff ANDREWS, 'Introduction', in Geoff ANDREWS, Citizenship, London: Lawrence \& Wishart, 1991, p. 12.

33. Laurent BOUVET, «Tony Blair est-il exportable? ", Politique internationale, $\mathrm{n}^{\circ} 80$, Eté 1998, p. 312.

34. Voir notamment, Amitaï ETZIONI, The Third Way to a Good Society, London: Demos, 2001.

35. Voir, à propos de ce courant essentiel du progressisme britannique, la riche étude de Michael Freeden, The New Liberalism : An Ideology of Social Reform, Oxford: Clarendon Press, 1978.

36. Le leader néo-travailliste promet en effet, dès juin 1997, que le New Labour soutiendra 'the thousands of social entrepreneurs [...] who bring to social problems the same enterprise and imagination business entrepreneurs bring to wealth creation' (Speech by the Prime Minister at the Aylesbury Estate, 2 June 1997).

37. Charles LEADBEATER, Civic Spirit: The Big Idea for a New Political Area, London: Demos, 1997, p. 11.

38. Charles LEADBEATER, The Rise of the Social Entrepreneur, London: Demos, 1997.

39. Ibid., p. 8.

40. Le travail de Putnam, très présent dans les écrits de nombre d'entrepreneurs intellectuels de la Troisième voie, transparaît régulièrement dans les discours de Tony Blair. Ce dernier défend en effet que le capital humain 'is at the core of the new economy. But increasingly it is also social capital that matters too - the capacity to get things done, to co-operate, the magic ingredient that makes all the difference. Too often in the past government programmes damaged social capital - sending in the experts but ignoring community organisations, investing in bricks and mortar but not in people' (Cité in Ian HARGREAVES, New Mutualism : In from the Cold. The Co-operative Revival and Social Exclusion, London: The Co-operative Party, March 1999, p. 6).

41. Marginalisé au sein du Parti travailliste en raison de son rejet de l'orthodoxie partisane, Frank Field a néanmoins fortement pesé sur le débat relatif à la réforme du Welfare que porte la Troisième voie. Nous nous permettons de renvoyer, pour plus de précisions quant aux facteurs expliquant l'importance de la contribution de Frank Field à ce débat, à notre article : «'Staying on benefits will no longer be an option': La Troisième voie et la théorie de la dépendance sociale " in Bernard LACROIX, Antonin COHEN \& Philippe RIUTORT, Qu'est-ce que l'analyse sociologique de la politique?, à paraître en 2005.

42. 'Long before the age of post-war State planning, and even Clause IV, British Socialists were popularly acclaimed for their identification with the principles of communitarian fraternity, mutual co-operation, individual responsibility and self-help. Co-operatives, friendly societies and trade-unions all formed an increasingly successful welfare network dedicated to the provision of [...] efficient [...] health and welfare 
services for their members' (Stephen POLLARD, Peter LIDDLE \& Bernard THOMPSON, 'Towards a More Co-operative Society : Ideas on the Future of the British Labour Movement and Independent Healthcare', Independent Healthcare, 1994, p. 1).

43. Edward THOMPSON, La formation de la classe ouvrière anglaise, trad. de l'anglais par Gilles Dauvé, Mireille Golaszewski et Marie-Noëlle Thibault ; présent. par Miguel Abensour, coll. « Hautes Études », Paris : Gallimard/Le Seuil, 1988 (1963).

44. Elles venaient ainsi en aide aux veuves et orphelins de leurs membres et disposaient de leurs propres médecins.

45. Gordon BROWN, ‘Civic Society in Modern Britain', op. cit.

46. Ian HARGREAVES, op. cit.

47. Les conservateurs emmenés par Margaret Thatcher entreprennent en effet, au travers de lois adoptées en 1980, 1982 et 1984, de réduire l'influence syndicale sur les relations industrielles en fragilisant durablement la solidarité entre les organisations et en en diminuant les ressources financières.

48. Peter MANDELSON \& Roger LIDDLE, The Blair Revolution : Can New Labour Deliver?, London: Faber and Faber, 1996, p. 226.

49. En 1984, la National Union of Mineworkers, dirigée par Arthur Scargill, entame une grève contre la Direction nationale des Charbonnages. Elle se solde, au terme de douze mois, par un échec en termes de revendications et par la scission du mouvement syndicaliste en deux à la demande des mineurs non grévistes. Fortement hostile aux grèves ayant marqué, à la fin des années 1970, le “ Winter of Discontent', l'opinion publique s'oppose, en majorité, à l'attitude adoptée par les organisations syndicales au cours de ce nouveau mouvement. Ce rejet des syndicats par une majorité des électeurs oblige dès lors le Parti travailliste à prendre ses distances vis-à-vis des premiers.

50. Nigel ASHFORD, 'New Labour's Hidden Agenda : Tony Blair's Plan to Privatise Health and Welfare', London: Libertarian Alliance, Political Notes, $n^{\circ} 136,1997,11 \mathrm{p}$.

51. Anthony GIDDENS, 'Brave New World : The New Context of Politics' in David Miliband, Reinventing the Left, op. cit., p. 33.

\section{RÉSUMÉS}

Cet article entend souligner que le mouvement de décentralisation conduit par le New Labour s'appuie sur des principes essentiels du projet politique néo-travailliste qu'incarne la «troisième voie ». Ses partisans entendent ainsi fonder une nouvelle relation entre les individus, les communautés et le gouvernement par le biais d'une valorisation de l'individualisation des sociétés, d'une nouvelle conception de l'égalité et de la constitution d'une « société civique ».

This article intends to emphasise that the movement of decentralisation pursued by New Labour rests on essential principles at the core of the New Labour political project: the Third Way. Its supporters seek in this way to build a new relationship between individuals, communities and government through the enhancement of a new individualism, a new conception of equality and the constitution of a 'civic society'. 
AUTEUR

JÉRÔME TOURNADRE-PLANCQ

Universités de Paris I (CACSP) et d'Orléans 